Praveen Chahar, MD, FCARCSI

Department of General Anesthesiology,

Anesthesiology Institute, Cleveland Clinic
Sidharth Dugar, MD

Department of Pulmonary Medicine,

Respiratory Institute, Cleveland Clinic
Donn Marciniak, MD

Department of Intensive Care and Resuscitation,

Anesthesiology Institute, Cleveland Clinic

\title{
Airway management considerations in patients with COVID-19
}

\section{Posted May 12, 2020}

\begin{abstract}
Approximately $12 \%$ to $15 \%$ of hospitalized patients with COVID-19 infection require invasive ventilation via endotracheal intubation. The key to minimizing risk of disease transmission during this aerosol-generating procedure is careful preparation, which includes having necessary equipment and medications on hand as well as an experienced intubation team who can troubleshoot any unforeseen complications. Personal protective equipment should be donned and doffed in the presence of an assistant (ie, "buddy" system).
\end{abstract}

\section{BACKGROUND}

Acute hypoxemic respiratory failure or acute respiratory distress syndrome (ARDS) is a common and serious complication of COVID-19 infection. ${ }^{1}$ Approximately $12 \%$ to $15 \%$ of these hospitalized patients need invasive ventilation via endotracheal intubation. In retrospective studies of COVID-19 patients, the onset of respiratory distress occurred late (median of 6.5 days) after symptom onset but progressed rapidly to ARDS (median of 2.5 days). ${ }^{1-3}$ Timely intubation prevents oxygen debt by optimizing oxygenation and ventilation. ${ }^{4}$

Endotracheal intubation is a critical procedure in the management of unstable patients with COVID19. However, rapid decompensation and hypoxemia have been observed during intubation from pulmonary derecruitment. Intubation can be associated with hemodynamic compromise from sympatholysis, vasodilation, and myocardial depression caused by induction agents. Intubation is an aerosol-generating procedure and is associated with a high risk of trans-

The statements and opinions expressed in COVID-19 Curbside Consults are based on experience and the available literature as of the date posted. While we try to regularly update this content, any offered recommendations cannot be substituted for the clinical judgment of clinicians caring for individual patients.

doi:10.3949/ccjm.87a.ccc033 mission to health care workers. Of all aerosol generating procedures, tracheal intubation is most consistently associated with disease transmission with an odds ratio of 6.6 and an absolute risk increase between $10 \%$ and $15 \% .^{5}$ Adequate preparation is paramount for the safety of the patient and providers. Table 1 outlines the key steps for health care providers to take before, during, and after intubation.

\section{PREINTUBATION CONSIDERATIONS}

The indications for intubation in patients with respiratory distress due to COVID-19 are similar to those in patients without the infection. However, patients with COVID -19 tend to deteriorate rapidly, warranting timely intubation. ${ }^{6}$ Ideally, patients are intubated in a controlled environment, such as an intensive care unit, but this may not always be feasible, and such a scenario should be anticipated. Noninvasive ventilation is associated with increased risk of aerosolization and should be avoided when transporting patients.

According to guidelines from the Centers of Disease Control and Management, appropriate personal protective equipment (PPE) should by donned before airway management ${ }^{7}$ in the presence of an assistant (buddy system) to ensure proper protocol is followed. Only health care providers directly involved in the intubation procedure should enter the room. Extra providers (runners) can be stationed outside to assist if needed. This is of utmost importance to minimize the transmission of infection. Ideal teams typically include a physician performing the intubation with a skilled assistant, a registered nurse, and a respiratory therapist.

An intubation bag containing medications required for intubation, appropriate vasopressors, and post-intubation sedative infusions should be brought into the room. To address the risk of sudden decompensation during intubation, a defibrillator and 'code box' containing resuscitative medications should be 
TABLE 1

Guidelines for performing endotracheal intubation in patients with COVID-19

\section{Preparation}

- Hand hygiene

- $\quad$ Full PPE per guidelines from CDC; use buddy system

- Team member outside room to help (full PPE)

- Prepare medications outside room

- Have ventilator available in room prior to intubation

- Reliable intravenous access

- Optimize patient position for intubation

- $\quad$ Airway assessment

- If difficult airway, have a team capable of performing emergency surgical airway available

\section{Equipment needed in room}

- Endotracheal tube (size 7.5 or $8 \mathrm{~mm}$ ) with stylet

- Video laryngoscope

- $\quad$ LMA size 4

- Intubation bougie

- Magill forceps

- HME/HEPA filter

- Capnograph

- Suction

- Endotracheal tube holder

- 10 cc syringe

- Fluid bag

- Barrier device
Intubation

- Preoxygenation with $100 \%$ non-rebreather mask for 3 to 5 minutes

- Do not initiate noninvasive ventilation

- $\quad$ Rapid sequence intubation; avoid bag mask ventilation

- Attach HME filter immediately after intubation

- Avoid auscultation to confirm endotracheal tube position

- Confirm tube position with inline capnograph

- If difficulty encountered, use supraglottic airway device (LMA)

Intubating medications

Induction agents

a. Propofol 1 to $2.5 \mathrm{mg} / \mathrm{kg}$

b. Etomidate 0.2 to $0.3 \mathrm{mg} / \mathrm{kg}$

c. Ketamine 1 to $2 \mathrm{mg} / \mathrm{kg}$

d. Methohexital 1 to $2 \mathrm{mg} / \mathrm{kg}$

Neuromuscular blocking agents

a. Succinylcholine 1.0 to $1.5 \mathrm{mg} / \mathrm{kg}$

b. Rocuronium $1.2 \mathrm{mg} / \mathrm{kg}$

\section{Initial ventilator settings}

- Pressure-regulated volume control with tidal volume 6 to $8 \mathrm{~mL} / \mathrm{kg}$ of ideal body weight

- Plateau pressure $<30 \mathrm{~cm} \mathrm{H}_{2} \mathrm{O}$

- $\mathrm{FiO}_{2} 1.0\left(\mathrm{FiO}_{2}\right.$ should be titrated to $\left.\mathrm{SPO}_{2}>92 \%\right)$

- Respiratory rate 12 breaths per minute

Postintubation

- Doffing per CDC protocol (use buddy system)

- Order chest radiograph

- Consider placing orogastic tube and invasive lines (eg, arterial and central venous pressure lines)

CDC = US Centers for Disease Control and Prevention; HEPA = high efficiency particulate air; HME = heat and moisture exchanger; LMA = laryngeal mask airway; $\mathrm{PPE}=$ personal protective equipment

stationed outside the room. There should be a ventilator available in the room so that patients can be promptly connected to minimize aerosol generation.

Intubation should be performed by the most experienced provider utilizing a video laryngoscope to minimize intubation time and the number of attempts. Use of a video laryngoscope versus a direct laryngoscope is associated with an increase in the distance between the health care worker and patient and thus may minimize the chances of cross infection. We recommend using a size $7.5 \mathrm{~mm}$ endotracheal tube for females and size $8.0 \mathrm{~mm}$ for males. Use of smaller sizes is associated with difficulty in ventilation and an increased likelihood of endotracheal tube obstruction with secretions.

Endotracheal tube obstruction caused by secretions is a common issue in patients with COVID-19 patients and may partly be related to the length of intubation as well as infrequent suctioning due to fear of contamination. Timely suctioning guided by the presence of secretions and the use of a humidifier can help prevent tube obstruction, which may require 
emergency tube exchange.

Airway equipment in the room should include a supraglottic airway device (eg, laryngeal mask airway or i-gel), gum-elastic bougie, and Magill forceps. Suction should be connected and be easily accessible to prevent aspiration once protective laryngeal reflexes are lost by induction medications. A reliable intravenous access should be established with a bag of intravenous fluid attached and running. Intubating conditions should be optimized, including bed height and patient position. All equipment including endotracheal tube should be pre-checked. A high efficiency particulate air (HEPA) or heat and moisture exchanger (HME) filter should be in the room with inline waveform capnography. If the patient has history of difficult intubation or if there are any signs of potential difficult airway, a team capable of performing emergency surgical airway in the event of difficult intubation and ventilation scenario should be available.

\section{INTUBATION PROCEDURE}

The aim of intubation is to rapidly secure the airway and minimize aerosol generation. Use of barrier devices, such as screens and intubation boxes (Figure 1), should be considered to prevent cross infection during intubation. ${ }^{3}$ A plastic sheet can be used if a screen and intubation box are not available.

Preoxygenation is best achieved by using a $100 \%$ non-rebreather mask on the patient for 3 to $5 \mathrm{~min}$ utes. ${ }^{4}$ Bag mask ventilation increases the risk of cross infection and should be administered only if absolutely necessary. Likewise, non-invasive ventilation should be avoided as a mode of preoxygenation due to concern of aerosol generation. However, if the patient is already on non-invasive ventilation, it should be continued. ${ }^{8}$

Short-acting opioids such fentanyl may induce coughing and should be avoided. Etomidate ( 0.2 to $0.3 \mathrm{mg} / \mathrm{kg}$ ) can be used in hemodynamically unstable patients or propofol can be used as induction in hemodynamically stable patients. Other induction agents include ketamine and methohexital. Muscle relaxation is achieved by either rocuronium $(1.2 \mathrm{mg} /$ $\mathrm{kg}$ ) or succinylcholine (1.0 to $1.5 \mathrm{mg} / \mathrm{kg}$ ).

Once the endotracheal tube is placed, it should be immediately connected to the ventilator/bag with an HME filter. The tube position can then be confirmed with tracing from inline waveform capnography. ${ }^{2,4}$ Breathing circuit disconnections should be avoided, and endotracheal tube clamping should be considered to prevent derecruitment and cross infection.

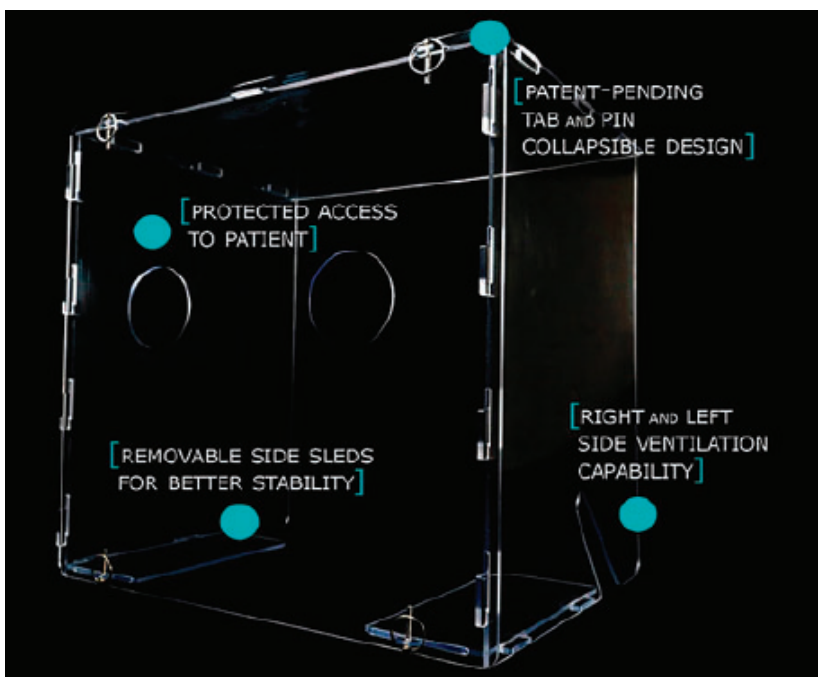

Figure 1. The AERO GUARD (C2Dx) barrier device.

If any difficulty is encountered during intubation and ventilation is required, a supraglottic airway device should be placed to help in oxygenation and ventilation in preference to bag mask ventilation. When using a supraglottic device airway filter (HME, HEPA), it should be placed between the supraglottic device and Ambu bag. Due to risk of aerosolization, fiberoptic intubation is discouraged and if needed, a disposable bronchoscope should be used.

\section{POSTINTUBATION}

We suggest initial ventilator settings as:

- Pressure-regulated volume control with tidal volume 6 to $8 \mathrm{~mL} / \mathrm{kg}$ of ideal body weight

- Plateau pressure $<30 \mathrm{~cm} \mathrm{H}_{2} \mathrm{O}$

- $\mathrm{FiO}_{2} 1.0\left(\mathrm{FiO}_{2}\right.$ should be titrated to $\left.\mathrm{SPO}_{2}>92 \%\right)$

- Respiratory rate 12 per minute.

Intravenous sedation should be started after intubation; choices include propofol and dexmedetomidine. Invasive lines can be placed after intubation if needed to reduce healthcare exposure and the need for additional radiographs beyond a chest film. All non-disposable equipment should be thoroughly cleaned according to infection control guidelines. Doffing and hand hygiene should be performed using a buddy system.

\section{REFERENCES}

1. Wang D, Hu B, Hu C, et al. Clinical characteristics of 138 hospitalized patients with 2019 novel coronavirus-infected pneumonia in Wuhan, China. JAMA 2020; 323(11):1061-1069. doi:10.1001/ jama.2020.1585

2. Yang $\mathbf{X}$, Yu Y, Xu J, at al. Clinical course and outcomes of critically ill patients with SARS-CoV-2 pneumonia in Wuhan, China: a singlecentered, retrospective, observational study. Lancet Respir Med. 
Published online Feb. 24, 2020. doi:10.1016/\$2213-2600(20)30079-5

3. Canelli R, Connor CW, Gonzalez M, Nozari A, Ortega R. Barrier Enclosure during Endotracheal Intubation. N Engl J Med. Published online April 3, 2020. doi:10.1056/nejmc2007589

4. Shoemaker WC, Appel PL, Kram HB. Role of oxygen debt in the development of organ failure sepsis, and death in high-risk surgical patients. Chest 1992; 102(1):208-215. doi:10.1378/chest.102.1.208

5. Tran K, Cimon K, Severn M, Pessoa-Silva CL, Conly J. Aerosol generating procedures and risk of transmission of acute respiratory infections to healthcare workers: a systematic review. PLoS One 2012; 7(4):e35797. doi: 10.1371/journal.pone.0035797
6. Meng L, Qiu H, Wan L, et al. Intubation and Ventilation amid the COVID-19 Outbreak: Wuhan's Experience. Anesthesiology. Published online March 26, 2020. doi: 10.1097/ALN.0000000000003296

7. Use of Personal Protective Equipment (PPE) When Caring for Patients with Confirmed or Suspected COVID-19. Centers for Disease Control and Prevention. WWw.cdc.gov/coronavirus/2019-ncov/ downloads/A_FS_HCP_COVID19_PPE.pdf. Accessed May 7, 2020.

8. Baillard C, Prat G, Jung B, et al. Effect of preoxygenation using noninvasive ventilation before intubation on subsequent organ failures in hypoxaemic patients: A randomised clinical trial. Br J Anaesth 2018; 120(2):361-367. doi: 10.1016/j.bja.2017.11.067 